Inan Klajn, Beograd

\title{
CARDUCCI E II LINGUAGGIO POETICO TRADIZIONALE
}

È indubbio che Giosue Carducci si trova a una svolta nello sviluppo del linguaggio poetico italiano. Prima di lui, alla conclusione del regno più che quadrisecolare della lingua petrarchesco-bembiana, i primi romantici avevano proclamato la necessità di. rinnovare, fra l'altro, anche il linguaggio della poesia. Ma il peso della tradizione era troppo grande perché essi, con le loro modeste personalità poetiche, potessero attuarè un vero sovvertimento. Il Berchet adoperò qualche parola non ammessa nel lessico poetico più tradizionalista, ma il fondo del suo linguaggio rimase decisamente aulico, con speme, desire, pièta, veglio, assiso, diè, sieno, fea, mancâr, seguio, rimanmi, nosco, il difende, ei preferse e simili anticaglie. ${ }^{1}$ Nel Prati, gli elementi nuovi stonano ancora di più contro lo sfondo tradizionale; ${ }^{2}$ l'Aleardi, poi, «segna un netto ritorno alla tradizione». ${ }^{3} \mathrm{La}$ generazione successiva al Carducci, invece, è dominata dal Pascoli, nella cui poesia non rimangono che rare tracce degli arcaismi ereditati. Anche per ragioni cronologiche, quindi, calcolando una specie di media aritmetica, potremmo aspettarci di trovare nella lingua del maremmano una uguale mescolanza del vecchio e del nuovo. Basterebbe a garantircelo, da una parte la prepotente originalità del poeta, mal disposto a riprendere da chiunque modelli linguistici già pronti, dall'altra il fatto che la sua creazione di un linguaggio proprio e personale era

1 V. Elwert, W. Th., La crisi del linguaggio poetico italiano nell'Ottocento, in Saggi di letteratura italiana (Studien zu den romanischen Sprachen und Literaturen, Band III), Wiesbaden 1970, p. 112-113. Le «parole dell'uso comune» che l'Elwert trova nel Berchet non sono affatto rivoluzionarie: pioppi, stenti, collo, buio, preghiera, sposa, rossore, bacio, giorno, affetti; inoltre «termini tecnici moderni e medievali: bandiera, squadre, brando, tricolore, elmetto, coccardas. Cesare de Lollis (Berchet, in Saggi sulla forma poetica italiana dell'Ottocento, Bari 1929, p. 38-40) dice che la lingua del B. è «il più strano mostro che si possa immaginare», in cui «elementi vecchi e nuovi tornano ad agitarsi incompostamente», e riportando alcuni degli arcaismi sopra citati commenta: «Tutto codesto vecchiume è pel Berchet moneta corrente».

${ }^{2}$ De Lollis, op. cit., 55; Migliorini, B., Storia della lingua italiana, Firenze 1963, IV ed., 603.

${ }^{3}$ Elwert, op. cit., 114. 
fermamente, programmaticamente basata sul rafforzamento della tradizione classica.

Questa è infatti l'impressione che numerosi critici hanno ricavato dalle loro analisi della lingua carducciana. Giulio Bertoni la definisce «insieme antica e moderna», precisando che «le parole non sono nuove; non sono nuove le locuzioni, attinte spesso ai classici latini e italiani; ma è nuovo il timbro, è nuovo il linguaggio del poeta.» ${ }^{4}$ La tradizione linguistica,» osserva il Devoto, «è per il Carducci, a p e r ta. È aperta non soltanto alle parole ornanti, tradizionali, che, data la sua preparazione letteraria, non sorprendono. È aperta ai neologismi, ai dialettismi, ai barbarismi, ai volgarismi».5 Il Paparelli dimostra che il C. è ugualmente lontano dai romantici e dai classicisti, dal manzonismo e dalla tradizione accademica, in quanto riconcilia $i$ poli apparentemente opposti della lingua d'arte e della viva parlata toscana. ${ }^{6}$ Il De Lollis trova nel nostro «i più forti arcaismi lessicali, morfologici, sintattici... nella coniugazione si direbbe che le forme arcaiche o poetiche prevalgono addirittura sulle normaTi», ma anche espressioni volutamente moderne, quasi giornalistiche, in una forma fonetico-metrica non ammissibile nella poesia tradizionale. ${ }^{7} \mathrm{E}$ molti citano l'ambigna dichiarazione dello stesso poeta: "Odio la lingua accademica che prevalse in molte opere poetiche degli ultimi secoli; ma amo, adoro la lingua di Dante e del Petrarca, la lingua de' poeti popolari del quattrocento, la lingua degli elegantissimi poeti del cinquecento, la lingua de' poeti classici dell'ultima età» (Ed. nazionale, vol. XXIV, 257).

In realtà è molto difficile dare un giudizio sulla novità o meno della lingua del Carducci. Se partiamo dall'impressione generale lasciataci da una lettura estensiva delle sue poesie, siamo tentati di dire che questa lingua è, sì, molto più viva e moderna di quella dei suoi predecessori. Ma appena cominciamo a sfogliare il testo, scopriamo con sorpresa che non c'è aulicismo o arcaismo poetico che non vi ricorra praticamente a ogni pagina. Si potrebbe osservare, e giustamente, che il linguaggio di un poeta non è uguale alla somma dei morfemi e dei semantemi adoperati, che esso è inscindibile dallo stile, dal pensiero e dalla forma poetica in genere. Ciò nonostante ci è parso utile eseguire un'analisi puramente linguistica della poesia carducciana, una specie di inventario che riunisca in forma sintetica e schematica tutti gli elementi derivati dalla tradizione aulica. Tale metodo ovviamente non pretende di fornire una soluzione

4 Bertoni, G., La lingua poetica di Giosue Carducci, nel vol. collettivo Carducci, discorsi nel centenario della nascita, Bologna 1935, p. 96 e 101.

- Devoto, G., Giosue Carducci e la lingua italiana, nel vol. collettivo Carducci, discorsi nel Cinquantenario della morte, Bologna 1959, p. 238.

- Paparelli, G., Carducci e il Novecento, Napoli 1953, p. 33-45.

7 Op. cit., 107-108 e 111-112. Già prima dí tutti questi critici, un analogo giudizio è stato espresso dal Pascoli: «Carducci ha dato all'Italia nuova la sua nuova lingua, portentosa, né aulica né volgare, e l'uno e l'altro» (Prose, ed. Mondadori, 1952, vol. I, 412). 
completa, ma siamo convinti che delle due facce del problema, esso possa rischiararne almeno una.

Trattandosi di un periodo relativamente vicino a noi, della cui lingua abbiamo numerosi documenti non solo letterari e nel quale la prosa, almeno, si avvia decisamente verso l'espressione realistica e moderna, non è difficile fare questa distinzione fra «arcaico» e «nuovo», sia nel lessico che nella morfologia. ${ }^{8}$ Tuttavia la nostra analisi resterà forzatamente incompleta sotto almeno due riguardi. Primo, non potremo fare altro che un breve accenno alla sintassi e all'ordine delle parole, che richiederebbero una trattazione particolareggiata e un metodo specifico. Secondo, in un poeta multiforme quale il C. sarebbe necessario distinguere fra gli aulicismi ormai assimilati, divenuti parte integrante del suo linguaggio, senza i quali egli non avrebbe potuto cantare, e le altre espressioni letterarie usate a scopi arcaicizzanti, per evocare un'epoca o uno stile, un preciso «color del tempo»: «parole poetiche» le prime, «parole storiche» le seconde. Questa ultima funzione degli arcaismi è facilmente individuabile nelle poesie giovanili scritte alla maniera degli stilnovisti, p. es. Questa è l'altera gioninetta bella / che tragge seco onesta leggiadria, ${ }^{9}$ ma la distinzione diventa quasi impossibile con la poesia satirica dei Giambi ed epodi ${ }^{10}$ o con le creazioni del poeta-vate degli ultimi anni. L'unica cosa che possiamo affermare con certezza è che nelle poesie liriche di argomento personale gli arcaismi «storici» sono assenti; purtroppo il limite tra queste poesie e le altre diventa sempre meno preciso

8 Mancano purtroppo una grammatica e un vocabolario del linguaggio poetico aulico, lacuna che ci proponiamo di colmare in parte in un altro lavoro. Per ora non ci sono che le brevi indicazioni in Migliorini-Chiappelli, Elementi di stilistica e di persificazione italiana. Firenze 1958, VIII ed., 119-132, e la lista di esempi lessicali nell'opera citata di Elwert.

${ }^{9}$ Questi versi sono citati dal Bertoni (op. cit. 90), che offre anche esempi del Carducci petrarcheggiante (né b'invidio, beati, il paradiso...) e foscoleggiante (qui done irato, agli anni tuoi novelli, / sedesti a ragionar col tuo dolore...). Si vedano anche le citazioni quasi letterali di Dante, del Tasso, del Foscolo e di altri, trovate nei testi carducciani da Antonio Baldini (Fine Ottocento, Firenze 1947, p. 256-259). «Era talmente satura», dice il Baldini, «la memoria del poeta, di tutta la poesia italiana, che magari inconsciamente se ne ritrovava $i$ segni sotto la penna più corrente».

${ }^{10}$ La poesia «giambica» presenta anche questa particolarità: che l'intenzione satirica vi agisce allo stesso tempo come elemento innovatore e arcaicizzante. Da una parte, questo genere di poesia presuppone l'uso di espressioni attuali e realistiche, che secondo il De Lollis cozzano «col fondo aulico della forma, rigorosamente aulico nel Carducci» (op. cit., 119); d'altra parte lo stesso principio di tale uso, essendo tradizionale, rappresenta per il C. un ritorno ai modelli berneschi e popolari toscani (si veda Borlenghi, A., Le ragioni della crisi delle forme e del linguaggio nella poesia italiana dell's00, Milano 1969 , p. CVIII-CIX). Pì̀ o meno simili ambivalenze si rispecchiano anche nei contrastanti giudizi sulle Odi barbare, la cui lingua secondo il De Lollis è tutta «schifiltosa, distinta, schiva d'ogni valore realistico» (p. 134), mentre l'Elwert, polemizzando col suo predecessore, vi trova «una forte venatura di linguaggio realistico» (op. cit., 124 ss.). 
nelle ultime raccolte, ed è tipico del C. il gusto di introdurre anche in una lirica personalissima, quale Idillio maremmano o Davanti San Guido, frecciate polemiche contro l'Italia del suo tempo.

Non abbiamo creduto necessario fare una completa statistica degli arcaismi lessicali e morfologici del C., quasi una «concordanza» delle parole adoperate. (Tale lavoro, tuttavia, se qualcuno potrà dedicarvi il tempo e lo sforzo necessari, risulterebbe certamente molto utile, in quanto la precisa cronologia fornita per tutte le poesie nell'Edizione nazionale permetterebbe di stabilire con matematica certezza le variazioni nell'uso dei singoli tratti arcaici o moderni della lingua carducciana.) $\mathrm{Ci}$ siamo invece limitati a registrare un certo numero di esempi per ogni parola o particolarità grammaticale discussa, sforzandoci di mantenerlo il più possibile proporzionale, cioè pari a una frazione costante del numero totale, in modo da offrire un'indicazione della frequenza relativa del fenomeno in questione. $\mathrm{Ci}$ siamo serviti dei primi quattro volumi dell'Edizione nazionale, indicando per ogni citazione le iniziali del titolo della raccolta e la rispettiva pagina del libro. Si ha così: $\mathrm{RSM}=$ Rime di San Miniato (vol. I, p. 5-103); PV = Poesie varie (vol. I, 331-466); $\mathrm{J}=$ Juvenilia (vol. II, 3-241); LG = Levia gravia (vol. II, 281-374); $\mathrm{GE}=$ Giambi ed epodi (vol. III, 3-132); RN = Rime nuove (vol. III, 159-370); $\mathrm{OB}=$ Odi barbare (vol. IV, 5-147); RR = Rime e ritmi (vol. IV, 175-255). Non abbiamo tenuto conto delle poesie stravaganti, rimaste al di fuori delle raccolte (si tratta per lo più di poesie giovanili, già sufficientemente rappresentate nelle prime tre raccolte).

I dittonghi mobili permangono ancora in forme arcaiche quali pruopa (RSM 32, LG 368, GE 23, RN 345), cuopre (GE 36), scuopri, scuopre (OB 12, 129), priega (RSM 56, PV 417), siegui (RSM 56, PV 449), niega, niegano (RSM 73, J 219, GE 22).

La monottongazione di uò, che può essere un arcaismo ma anche un toscanismo, è molto frequente: novo (RSM 35, 90, J 14, 233, LG 316, RN 168, 329, RR 183, 243, 245: ma nuobo in RN 271, 317, OB 118 e altrove); move, mova, rimove (RSM 14, 74, LG 282, RN 249; ma muova in PV 392 e altrove); more, mora (RSM 18, 36 ecc., ma muore in LG 48, RN 328); scota, scote, scoter (RSM 56, 99, RN 195 ecc., ma scuota, scuote e perfino scuotesti in $\mathrm{GE} 73, \mathrm{OB} 20,114$ ecc.); percote ( $\mathrm{PV} 368$, ma percuotere OB 129); foco (LG 335); occhiaie pote (LG 332). Le forme cor(e) e cuor(e) si alternano costantemente, senza alcuna regola, lungo tutta la produzione carducciana: la prima in RSM 6, 17, 27, 51, PV 334, 345, 366, 400, 449, J 13, LG 281, 301, GE 4, 51, RN 206, 342, 359, OB 123, RR 175, 255 ecc.; la seconda in RSM 45, PV 381, LG 296, 305, 308, 349, GE 17, 28 , 115 ss., RN 170, 188, 281, OB 24, 60, 131, RR 180, 211, 247 ecc. Esse si incontrano anche all'interno di una sola poesia (p. es. "Rimembranze di scuolas, RN 266 e 267) o in due strofe consecutive (p. es. RR 211). In 
questa incostanza nell'uso del dittongo il C. può aver seguito l'esempio del Petrarca o di Dante. ${ }^{11}$

Rarissima invece la monottongazione di iè: poco mèle (PV 465).

Le forme $d e i$, ai, ecc., davanti ad altre finali in $-i$, sono normalmente apocopate (come, del resto, in gran parte della prosa dell'Ottocento): co' tempi (RSM 15), de' tuo' amori (36), l'aurea / giovenil chioma e' rai (80), tra' servi (83), que' marosi ( $\mathrm{PV}$ 335), tu se' schiaoa (350), be' pensier' d'amore (401), tra' lirici (J 3), be' circoli (162), ne' bei piani (LG 289), pe' campi e ne' verzieri (307), da' vetri (358), da' preti (GE 106), a' suoi be' dì (107), contro i servi e' tiranni (RN 166), dé miei sogni (197), pe' verdi colli, da'cieli splendidi, / e ne' fiorenti campi (OB 116). Ma il Carducci, a differenza della poesia tradizionale, usa questa apocope anche alla fine del verso: oh qual tu se'? (LG 302), un pover uom tu se' (Davanti San Guido), entrambe le volte in rima con me.

Il troncamento in fin di verso è una caratteristica della poesia ottocentesca $^{12}$ e in specie di quella carducciana, a cui spesso dà un'aria di canzonetta, con rime come fior : amor (Pianto antico), filar : guardâr (D. S. Guido), padron : ciceron (GE 10), destrier : fier (59) assal : Quirinal (97) ecc.: si veda, per mna serie di esempi, l'intera poesia In Carnia, RN 239-241. È normale il troncamento di - $i$ : battaglier morenti (RSM 44), gli error cari (PV 461), i sentier tacenti ( $\mathrm{J}$ 19), il nome de i maggior (214), la terra germoglia guerrier (236), $i$ color (LG 282), i fior (310), astieni, astien la vergine / man (341), doman ( $\mathrm{GE} \mathrm{45),} \mathrm{vision} \mathrm{leggiadre}$ (RN 174), dormono i cuor (OB 89). Si tronca anche il pronome affisso lo: tenetel chiuso ( $\mathrm{J} 161$ ), portal con suono (GE 8), scossel (41) rendimel (RN 349), ponendol (OB 21).

Frequenti le elisioni, soprattutto dell'aggettivo preposto: italic'arte (RSM 12), mitic'oro (85), tropp'alto (LG 354), l'attonit'onde (GE 7), lasciatem'ire (RN 281), rigid'aere (OB 112). Si elide, beninteso, anche l'articolo femminile plurale: l'ombre, l'erbe, l'aure (RSM 24), l'arti (RSM 91 . $\mathrm{PV}$ 355), l'iberiche fiamme (J 6), l'ulcere (GE 122), l'anime (OB 121).

Appaiono quasi tutte le apocopi tradizionali: ve' per vedi (RSM 12, 49, PV 336), diè per diede (RSM 38, 50, PV 356, 376, J 163, 226, RN 166. 340, RR 187), fé per fede (LG 337, GE 42, RN 289), piè per piede (RSM 95, PV 347, J 207, GE 12, RN 175), vo' per voglio (PV 399, 465, GE 3, 8, 63, RN 177, 219, 360, RR 212, 235), pèr per verso (IG 284, 368, GE 102, RN 280, OB 23, 53, 111). Cf. e sempre al solito mo' tolleranti, J 6.

Lo stesso per le sincopi: opra, oprare (RSM 53, LG 357, RN 172, 188, 272, OB 12, 73, 124), carco, scarco (RSM 8, PV 373, LG 355, 367, RN 305), se ne rammarca ( $\mathrm{RR} 247$, in rima), spirto ( $\mathrm{RSM} 23, \mathrm{PV} 343, \mathrm{GE} 68, \mathrm{RN}$

${ }_{11}$ V. Giannuzzi Savelli, F., Arcaismi nelle rime del Petrarca, in «Studi di filologia romanza», VIII (1899), a p. 94-97; Migliorini, Storia, cit., 206; Schiaffini, A., Le origini della lingua poetica italiana, Roma 1940, parte IV, 70-71.

12 Elwert, op. cit. 123-124. 
172, OB 82, RR 180), dritto per diritto (PV 445, LG 304), biasmo (RSM 12), diciottesmo (PV 429), e il meno solito scheltri (GE 43, 44, 99).

Poco frequente la sincope dei verbi in -gliere: scêrre (PV 409), sciôr (GE 101, 130), accôrre (RP 222).

Tra le aferesi, quella di il ha un significato soprattutto grafico, dato il vezzo carducciano di scrivere staccate le preposizioni articolate ( $s u$ ' $l$ e simili). Ma troviamo anche tra $i$ monti e ' $l$ mar ( $\mathrm{J} 207$ ), qui 'l fresco, qui 'l sonno (OB 57), e nelle poesie giovanili anche umana industria 'n divo lume abvolta (RSM 85), e'n lui tutto versando (PV 337), d'ogni gioia ' $n$ bando (383), de l'itala famiglia 'nfami ludi (355), e 'nvitto e forte (375), 've allettano i Piacer con riso infido (RSM 335), là 've la sulfurea notte alberga (PV 426).

L'i prostetica è più frequente nelle poesie giovanili: chiuso ne le spelonche isbigottioa (RSM 94), accorrenti irrompenti isplendienti (99), una immagin di donna isconsolata (PV 459), in lusinga serbile iscibolò (GE 43). Raramente il C. se ne serve a scopi metrici: con ischietti d'amor cenni e parole (LG 295), iscaturiscan di fumoso oin (GE 6), ed isgrammaticate (settenario in GE 93).

I sostantivi tronchi mantengono spesso la sillaba finale, sia -te che -de: beltade (RSM 7), ree volontadi (11), felicitate (90), pietate (PV 386), gioventude in rima con virtude (420), etate i.r. c. beate (LG 284), egualitade (LG 348, RR 196), falsitadi (GE 54), beltate i. r.c. vate, alate, fate (RN 182), cittadi (RN 216, OB 14, RR 242), nobiltade (RR 228), ecc.

Si usa ancora quai per quali (quai d'ozio promesse, RN 170, quai nel pensier... / $i$ dolci anni perduti, 211, quai canti, OB 94), e quei per quello o quelli: quei che il paradiso vide (RSM 45), quei che forti / furo (95), quei le livide note / mostran (LG 332), quei che a te niegan la patria (GE 22), quei che ben la sa (RN 348), quei che anelando vittoria caddero (OB 73), quei che a Sfacteria dorme (RR 187). Appartengono allo stesso tipo fonetico le forme augei (LG 309, i. r. c. imenei, GE 26, RN 198, 210) e capei (capei grigi, GE 99). Per ei = egli v. più sotto.

Frequente -gn- per -ng-: giugnea (RSM 21), piagne (RSM 23, J 175, LG 325, 358), strigne ( $\mathrm{PV} 406$ ), io vegno (GE 49, in rima con sdegno; $\mathrm{RN}$ 181 , i. r. c. ingegno, sostegno, segno; $R R$ 236), rimagna ( $R N$ 317, i. r. c. Spagna), cignale (PV 403, GE 43, RN 273, OB 52, RR 223), ugna (RSM 72, GE 100; ma unghie RSM 94).

Raro invece l'altro arcaismo fonetico comune nella poesia tradizionale, -gli- per -lg-: raccoglia (RSM 7, i. r. c. spoglia), chi sa non l'assaglia / una deserta polontà d'amare (GE 112, i. r. c. Sinigaglia).

Dei verbi in -dere si ha (ri)veggo, (ri)pegga (usato dal C. anche in prosa: RSM 26, PV 341, 450, J 6, 200, LG 291, 361, RN 178, 197, GE 50, $\mathrm{OB}$ 14, RR 220; ma peggio in PV 433); peggendo (RN 266), seggo, segga (RSM 38, 46, RN 181, RR 247), chieggo, chieggono (RSM 28, 59, 100, J 37, LG 290, 299, RN 254, OB 103; ma chiedo, OB 137), caggio, caggiono (RSM 98, RR 211). 
In rima, e anche fuori, sono usate le tradizionali piane al posto di sdrucciole: umile (RSM 82, in rima, PV 345, 397, GE 21 i. r., RR 227), simile (OB 102), tenèbre (GE 12, i. r. c. funèbre, $\mathrm{PV} 394, \mathrm{RR} 228$ ), penètra (RR 230), intègro ( $\mathrm{PV}$ 370), edùca ( $\mathrm{PV}$ 347, GE 6), esplìca (LG 305, i.r.), oceàni (RSM 84, i. r.).

L'articolo lo per il è usato, secondo l'uso ancora prevalente nell'Ottocento, ${ }^{13}$ dopo per (per lo mar, RSM 41, per lo ciel, PV 388, per lo mondo, RN 227), e nelle poesie giovanili, come voluto arcaismo, anche in altre posizioni: lo ministro maggior de la natura (RSM 45), de lo amore ( $\mathrm{PV}$ $347,392)$, la valle de lo giglio (348), lo arciero Iddio (381), lo amico suolo, lo aratro (408), lo aspetto $(372,425)$, lo tuo regno (RN 316$)$.

L'articolo plurale $l i$ sostituisce $i$ dopo per (per li verdi tramiti, RSM 10, per li deserti, 41, per li colli, PV 403) e spesso si usa al posto di gli:14 li dèi (RSM 80), li ameni prati (PV 335), li atti (372), li sdegni (385; nello stesso verso gli amori), li scogli ( $\mathrm{J} 236$ ), li spiriti magni (OB 31), li occhi (61), li usignoli (79), li ardenti occhi (RR 239). Un caso eccezionale è per gli silenzi (LG 284).

Anche la licenza poetica di adoperare l'articolo $i$ davanti a $s$ impura non è sconosciuta al C.: de' scoppi (PV 428), tra i sparsi liguri (RR 192).

L'articolo determinativo o indeterminativo, secondo un modulo tipico della lingua poetica, viene liberamente omesso: non per cifre e teoremi acuti / d'economista (RSM 90), anima intègra da viziosa cura (PV 370), se losca Diva urge Fortuna ostile, / cuor preparato il suo cangiare attende (381), t'invita più benigno ardor di cielo / e primavera di straniero lido (J 14), da la madre battuto pargolo / od in proterva rissa mal domito (OB 108), come per sereno cielo / stella bolante (RR 223).

La forma debole di io appare soltanto nelle liriche giovanili (più ch' $i$ ' non vorrei, $\mathrm{PV} 464 ; i$ vi prego, J 161), mentre ei per egli è largamente usato (RSM 21, 87, PV 338, 421, 464, J 8, 161, 205, LG 312, GE 14, RN 172, $\operatorname{RR} 178,211,238$, ecc.), anche col valore plurale (ei con iscandalo ti buttan là, J 7, ei fûr che la vittoria / vi contesero, GE 36, ei fuggiranno, 80, ei dormon, RN 253, ei deliraro, OB 28, ei le braccia / al sole a i giuochi tendono e sorridono, $\mathrm{OB} 99$ ).

Vui per boi, rimato con lui e figli sui, appare in RSM 82; nel rifacimento dello stesso verso in J 135 al posto di oui si ha nui.

Le forme meco, teco ecc. sono usate con moderazione (meno, p.es., che nel ben più moderno Pascoli): l'alma teco in un disio congiunta (RSM 8), quando meco fremon gli op pressi (PV 385), ho con meco / tutta dolcezza (401), teco io m'affiso, / teco m'esalto (LG 293), verran teco (GE 8), poi nosco ti addurrem ( $\mathrm{RN} 255)$ seco piange (347), teco l'Erinni sale (OB 76).

Sull'esempio forse del Parini e di altri poeti latineggianti, i pronomi tonici sono volentieri usati al posto degli atoni: tu me reggi e affranca

13 V. Migliorini, Storia, 630, 705.

14 Ivi, 629, 704. 
(RSM 13), che te laudassim noi (16), qual sé levando / scotea dal capo del servaggio $i$ danni ( $\mathrm{J} 223$ ), tu lei togli a l'aspetto / ... de l'uno e l'altro amato suo parente (LG 313), io... / immortal lui credena (GE 90), april te vide su 'l colle emergere (OB 15), la bianca / uoa... / sé disfacendo il forte e redolente / vino matura ( $R \mathrm{R} 243$ ). Si trova anche lui con valore dativale: Lui dite: In sen de $i$ Versiliesi nidi... (PV 383).

Non rari i pronomi atoni arcaici $i l(=l o)$ e ne $(=c i)$ : il saprai (RSM 35), al patrio nido da cui lunge il colse (PV 383), la vista, che il circonda (LG 282), io 'l vidi (366), il pedess'io (RN 177), e il giungi (OB 12), tedio il consunse (RR 222); per te vita n'è spenta (RSM 53), diciam parole prospere: / benigno Amor ne appare (74), tu, o Signore, danne laboro (PV 408), ov'ella n'abbandona (LG 310), o testimone di tre imperi, dinne... (OB 25), eccoti il re, Signore, / che ne disperse, il re che ne percosse ( $R R$ 187).

Al posto del pronome atono di $3^{a} \mathrm{pl} . \mathrm{m}$. $l i$ si ha spesso gli:15 infondli a' cor di quel valore / che gli rapisca a più sublime sfera (J 103), gli attese al passo (206), io gli ho sepolti (GE 3), gli abbrancava e gli bollava in viso / e gli gettava ne la morta gora (RN 180), nel sol che gli penetra (RR 230).

Comunissima l'enclisi del pronome o della particella atona coi modi finiti: avoi (RSM 31), se pur prèmeti desio (49), erangli intorno (97), ponmi (PV 371), poserovvi (386), mille stannole attorno ombre (J 192), segnapanlo (LG 295), e cinselo e girossi (335), gridingli (350), dirògli (GE 16), vommene (124), rampolommi in cuore ( $\mathrm{RN} 266$ ), ricamòglielo (349), io còlgola (OB 5), perderannosi (50), chinommisi il capo (123), fino a che l'ora sacra richiamine (128), viensene Aroldo (RR 189), ecc. ecc. Con un avverbio: biancheggian teschi per le rupi orrende, / e soprabi la nera aquila posa (RN 167), e sóprabi giganti ( J 175).

Viceversa, ma meno spesso, si ha la proclisi del pronome con l'imperativo: deh! de 'l vero e di te m'apri la via ( $\mathrm{PV} \mathrm{331),} \mathrm{dove} \mathrm{regna} \mathrm{lo} \mathrm{stra-}$ niero / va', ti mostra ( $\mathrm{J} 217$ ), m'udite, o voi / che un di m'amaste (GE 55), portami... / ne $i$ campi de le stelle mi porta (OB 14), nuoola bianca, t'apri (RR 188).

Un isolato arcaismo è rappresentato dagli esempi levosse e calosse, rimati con mosse e commosse, in LG 366.

Le coppie di pronomi atoni possono ancora venire troncate: né se 'n piange il poeta (RSM 96), me 'n vo soletta (PV 401: il posto dell'apostrofo, in questi due esempi, è sbagliato ma abituale anche in altri poeti), no 'l so (429), te 'n preghiam (450), a te no 'l dissi (RN 221), peso d'oro te 'n vo' dar (349). L'ordine dei pronomi è quello oggi normale, ma troviamo anche questo raggio d'amor no 'l m'invidiate ( $R N 255) \mathrm{e}$, in una delle ultime poesie, noi la ti demmo (RR 249).

${ }^{15}$ Il Migliorini nota quest'uso soltanto per il primo Ottocento (Storia, 628). 
Unicamente nelle poesie giovanili arcaizzanti leggiamo le forme tui, sui (RSM 82, LG 323 due volte, sempre in rima) e suoi nel senso di loro (pietose / de i dolori non suoi piangean le spose, RSM 87).

Il pronome relativo cui è adoperato spesso con valore di accusativo: de' verd'anni amica / cui d'estri infiori e di dolcezze (PV 368), Gliceria... / cui le Grazie educaro (J 153), l'osseo petto cui la tosse scuote (LG 303), uomo cui molta birra gravi (OB 102), o al posto di chi: cui sposò la fanciulla? (PV 392), cui vi strappa de' vostri avi il retaggio, / cui vi tragge a servir, Dio non perdona (J 224), a cui la morte teme non ridono / le muse (OB 84).

Rari (mentre sono comuni nei poeti precedenti, fino a Leopardi compreso) i pronomi relativi che e chi usati con preposizioni: petto.../ in che il valor de' padri oggi sormonte (RSM 16), l'antica madre in ch'io mi vanto (J 191), ferocie vane / in che il tuo cor si esala (GE 32), le immagini de' grandi in chi s'aduna / quantunque è de 'l buon seme (RSM 54).

Vari verbi di stato, di movimento e altri, intransitivi oggi, hanno nel C. la tradizionale forma riflessiva: ${ }^{16}$ tale mi son... / io (PV 372), se tuoi doni si fûro (366), io non so che si sia (GE 109), o biondo siasi o nero (RN 207), con te si stette Amore (RSM 39), il mio cuor solo stassi (RN 170), e morirommi sola (PV 465), ei viene e si muor.../ ov'è che Giaufredo si muore? (RR 178), la commedia de l'arte si dormia (216), se m'ebbi alma da te (PV 366), il sole... / partendosi, la favolosa / cima fesulea tinge di rosa ( $\mathrm{J}$ 9), al loco onde si parte ogni possanza (LG 326), amor con li anni fuggesi (PV 422), fuggiasi anch'esso (LG 320), vedea/ quel corpo tenerello, e si piagnea (PV 464), ecc.

Verbi della III coniugazione privi dell'infisso -isc-: $e$ pèra il grave secolo (RSM 80), pur fia che pèra (PV 386), il fere / da l'anvenire un raggio (GE 47), del fulmin tutto dì mi fere ( $\mathrm{RN} 294)$, se... / cape ne' vostri angusti petti il duolo (J 13), ove non più si pate (141), flue l'onda innamorata (PV 404), quella età che non rinverde (LG 291), ecc.

La terza persona singolare del congiuntivo, e più raramente la $2^{\mathrm{a}} \mathrm{sg}$. dell'indicativo dei verbi della I coniugazione possono terminare in - $e$ anziché in $-i$ quando lo richieda la rima: sormonte (i.r.c. monte, fronte, conte, RSM 16), dèste (i. r.c. queste, $\mathrm{PV}$ 338), si raffronte (i. r. c. fronte, conte, monte, J 93), par che sepolcro al corpo vivo apporte (i. r. c. morte, consorte, scorte, LG 282), s'Alpe ed Ato pria non si distempre (i. r. c. tempre, $\mathrm{RN}$ 168), e par che sangue cole (i.r. c. sole, prole, 323); te ... / chiamo, te che da noi ti discompagne (i. r. c. campagne, RSM 10), te... che prove / sì degne mostri onde a ben far c'incore (i. r. c. onore, 56).

La prima persona dell'imperfetto finisce sempre in -a: io la mirava (LG 318), io ti seguia (GE 13), del quale udina io parbolo / mirabili parole (90), io memore sognava ( $\mathrm{RN} 260$ ), io così piangeva (284), te io boleoa

${ }^{16}$ Si tratta di un uso comune in tutta la poesia tradizionale fino a Manzoni e Leopardi, ma esemplificato, per ora, soltanto nei testi più antichi da Franca Brambilla Ageno, Il verbo nell'italiano antico, Milano-Napoli 1964, 136-148. 
(OB 77), io guardapa la madre (124). Una sola volta, in rima, appare la desinenza moderna: Era tanto che giaceoo! / E tornato il medio evo! (J 187).

L'imperfetto della II e III coniugazione è normalmente privo della -D- intervocalica: giacea, ardea, rompea, sorridean, porgean, sentia, fioria, copria, fuggiano. morian, ecc. Anche nella $2^{\text {a }}$ sg. si ha p.es. tu.../ dolo e disdegno avei di te (RSM 43), pia /avei l'alma (RR 233), tu dritto in piedi tutta / ergei la testa (LG 319). A volte però queste forme si alternano con le forme intere: a te cadeva il braccio, e ti battea / alto anelito i fianchi (RSM 99), saliano fiamme ch'astri parevano (OB 64), già ferveba fremeoa urtabasi (LG 361). Rara la desinenza -ieno per -iano (uscìeno, RSM 86, GE 50). Non si trova l'imperfetto in -ia, -iano dei verbi di II coniugazione (tipo abia, soliano).

Volentieri usato dal C., nonostante il suo carattere arcaico, è il passato remoto paragogico dei verbi della III coniugazione: $t i$ tradio (i. r. c. natio, pio, oblio, RSM 19), sentio (39), fuggio (58), impaurio / le vergini (PV 356), coprio i. r. c. ruggio (426), sentio e finio i.r. c. Dio, pio (450), fiorio (i.r. c. mio, $\mathrm{RN} 216$ ), muggio (OB 32), tutto panio (i. r. c. leggio, addio, RR 216), al sacro monte fue (236).

Nella $3^{a}$ persona pl. del passato remoto regolare è normale la desinenza -ro: spiraro (RSM 28), dilaceraro (93), $i$ miei canti... volâr; / $i$ fantasmi... favellâr (PV 342), che padri avventurosi / al secol ti donaro? / che tempi ti portaro / così bella? (457), ruinaro a la tenzon (J 210), m'abbandonaro (LG 284), sentîr l'arcana deità presente / le plebi (366), impallidîr le rose, / moriro $i$ sogni (GE 3), le nubi che grabârmi tanto (RN 184), balzâr nel buio (OB 61), le rose sfioriro (RR 218). Non appare invece, dopo il periodo giovanile, la desinenza -no per -ro: miei desiri / pace ebbon (RSM 10), se fussin soldi ( $\mathrm{J} 181$ ).

Le terze persone del condizionale hanno la desinenza -ia, -iano: tuo pianto destar la porria (PV 374), non altro a desïar lo spirto abria ( $\mathrm{J} 66$ ), darian (LG 292), vorrien (370), vorria (LG 284, GE 31, RN 277, 299), avria / aggiudicato (GE 68), faria (81), rifiorito... / saria l'aprile (RN 261). Rarissime le forme moderne: onor la brutta / schiera s'abrebbe ( J 89), gemerebber più dolci e l'aure e l'onde (LG 292).

Non molto usata la forma breve del participio passato dei verbi della I coniugazione: in fin che tempra umana / non sia da 'l vizio macerata e doma (RSM 11), il fortissimo amor / ch'oprando hai mostro (53), non compri baci, / liberi amplessi (PV 385), di ridesti popoli / fremon le valli ( $\mathrm{J} 226$ ), le tue valli non tocche (LG 326).

Del verbo essere, è comune il futuro fia (RSM 29, 54, 88, PV 334, 386, 458, J 214, LG 339, GE 8, RN 230, OB 76, 128, ecc.), pl. fiano (LG 321) o fieno (RSM 55, GE 38), la $3^{2} \mathrm{pl}$. del passato remoto furo, fûr ( J 199, GE 64, RN 217, 261, OB 19, RR 178, ecc.). Nel cong. pres. notiamo $3 \mathrm{pl}$. sieno (RSM 26, J 10; cf il secolo / sieci se vuol nimico, RSM 59). 
Forme arcaiche del verbo fare: $1^{\mathrm{a}}$ sg. del passato remoto fei ( $\mathrm{J} 123$ ), $2^{\text {a }}$ sg. festi (LG 350, RR 249), 3a sg. fe', feo (RSM 12, 89, J 209, GE 53, RN 179, 207, 281), $1^{\text {a }}$ pl. femmo (PV 411), 3a pl. fero (RSM 351). Anche l'imperfetto fea (gli fea catena, PV 423).

Verbo andare: infinito ire (LG 285, GE 109, RN 272, 281), gire (RSM 7, 89, PV 341, RN 227), imperativo ite ( $\mathrm{J} \mathrm{167),} \mathrm{imperfetto} \mathrm{iva,} \mathrm{ioano} \mathrm{(RSM}$ 101, LG 357, RN 298, OB 75, RR 248) o giva, gìa (PV 336, OB 79), imperf. cong. gissero (come pirati che a preda gissero, $\mathrm{OB} 68$ ), part. gito (dove se' gita, RSM 20; se n'è gita, J 117).

Verbo donere: $3^{\mathrm{a}}$ sg. pres. dee (RSM 12, PV 453, LG 350, GE 122, 130, RN 225, 315).

Verbo volere: $1^{\text {a }}$ sg. pres. $v o^{\prime}$ (citato sopra, fra le apocopi).

Verbo potere: $3^{\mathrm{a}} \mathrm{sg}$. pres. puote (PV 338).

Verbo dare: $3^{a}$ sg. pass. rem. diè (citato sopra, fra le apocopi), pl. diero (PV 398, 458, J 94, 235, RR 227).

La parola onde è ancora usata nei suoi significati poetici: avverbio interrogativo (onde penisti?, $\mathrm{OB} 78$ ), congiunzione consecutiva (e scioglie / dolce la pura angelica fabella: / onde ardo, e pace io non abrò più mai, RSM 25; Bavio t'odia, o sonetto; ond'io più t'amo, RN 165), pronome relativo, col senso di «di dove» (ne i campi de le stelle mi porta, ond'io vegga la terra, $\mathrm{OB} 14$ ) oppure «di cui, con cui» (quel flagello / onde me... / il disinganno sferza, RSM 9; co 'l dolore onde $i$ lassi occhi velo, $\mathrm{J} 14 ; i$ mostri onde tu... / farai franche le genti, OB 17).

Fra le congiunzioni, nótiamo l'uso di però che «perché»: $E$ degno è ben, / però ch'a te potei / chinar l'ingegno (RSM 26), cede il clamor bugiardo / al silenzio... / però ch'eterno il tuo foco s'accende (LG 335), però ch'io sono la gloria (OB 21).

Preposizioni antiquate: appo (appo una tomba, J148, appo l'onde, RN 287 appo $i$ clivi, OB 69), in su (in su i sentier, RSM 10, in su la sponda, PV 375, in su la sera, LG 311, in su l'aspro sentiero, GE 56, in su le piazze, OB 79), in fra (in fra gli allori, RSM 24, in fra $i$ vioenti, PV 369 , in fra gli abeti, RR 232), sott'esso (sott'esso il fasto de l'eretto ciglio, LG 295, sott'essa la tua funerea volta, GE 49, sott'esso il brando / di Rolando, RN 160), soor'esso (sovr'esso il mare, RR 236), lungh'esso (lungh'esso il fiume sacro, OB 31).

Le preposizioni articolate, com’è noto, sono dal C. scritte staccate, con qualche concessione all'uso moderno, soprattutto nella scrittura di al, del, nel. Una sola volta troviamo l'arcaico in le (ti specchi in le soavi acque tranquille, PV 346).

La sintassi del C. - astraendo per il momento dall'ordine delle parole - non ha nulla di particolarmente aulico, anzi a volte è decisamente popolaresca (meglio era sposar te, bionda Maria). Troviamo il condizionale semplice usato nella concordanza dei tempi con la funzione dell'odierno condizionale composto (sperai che ... / potrei ne' poti unanimi / seguir con l'inno alato / l'ascension, LG 347), ma è un uso ancora largamente 
prevalente nell'Ottocento. ${ }^{17}$ Le proposizioni concessive hamno ancora qualche volta il verbo all'indicativo: se bene ancora / lui la chiarissima viltade adora ( $\mathrm{J}$ 8), ben che la stanza mia qui sarà corta (RR 236). Relativamente frequente è l'accusativo alla greca: ed ei sparso di rigido / livor la bella faccia... ( ( 219), la vergin pallida /... / alta levossi, a gli omeri / lenta il crin biondo (LG 298), tu . . / affocata le guance . . . / corri (OB 12), il padre, di caprine pelli / l'anche rapoolto (23). Ma si nota già l'intrusione della moderna costruzione assoluta, come nel seguente passo in cui soltanto la terza proposizione contiene un vero accusativo alla greca: Date al vento le chiome, is f a $v i l l$ ant $i$ / gli occhi glauchi, del sen nud a il candore, ! salti su'l cocchio (LG 370).

L'ordinamento delle parole è meno libero, più lineare di quello di gran parte della poesia tradizionale. Piuttosto che un ordito sintattico vario e movimentato, troviamo a volte un'inattesa disinvoltura nella collocazione degli elementj della frase, come nei famosi cipressi che, anziché da San Guido a Bolgheri, vanno «a Bolgheri... / da San Guido». Rientrano nella sfera delle inversioni tradizionali esempi come su le paterne ossa giurato / questi ha il mio scampo ( $\mathrm{J} 205$ ), de' forti anni la virtù (GE 37), tu zampilli / su del popolo dal cuore (RN 159), il restio / seno a $i$ freni del vel (271), hanno / de l'adamante rigido i riflessi (OB 26). Un influsso del Parini e degli altri poeti settecenteschi cari al nostro si potrebbe ravvisare nella tendenza a staccare l'articolo e l'aggettivo dal sostantivo corrispondente, ad es. per la breoe che ho corso ispida via (PV 366), rivedrem le belle, / che ne disser piagnendo il lungo addio, $;$ facce d'amore (LG 325), e tutte il mare spinge le mugghianti / collere (OB 74), quella che sempre negaronmi i fati / pace d'affetti (OB 125), cercan le deste a ragionar di gloria / ville e cittadi ( $\mathrm{RR}$ 182). D'altra parte - e sebbene il nostro sia, anche in questo riguardo, molto meno innovatore del Pascoli -- tipica del Carducci maturo è la narrazione piana, diretta, senza trasposizioni. Quando essa è turbata, ciò non avviene, come in tanti altri poeti e nello stesso $\mathrm{C}$. giovane, unicamente sotto la sforzatura del metro e della rima, ma serve piuttosto a ottenere un particolare effetto ritmico-sintattico. Così, Traversando la Maremma toscana e Sogno d'estate, per citare solo due tra le poesie più famose, hanno un ordine talmente lineare che potrebbero essere trascritte in prosa quasi senza cambiamenti. In altre poesie, le uniche deviazioni dall'ordine normale sono costituite dai chiasmi (p. es. nel Pianto antico - tu fior de la mia pianta... / tu de l'inutil vita / . . fior e né il sol più ti rallegra / né ti risoeglia amor) o da altre, intenzionali e finemente architettate figure simmetriche (p. es. in Santa Maria degli Angeli - Oh che una traccia / diami il canto umbro de la tua parola, / l'umbro cielo mi dia de la tua faccia).

${ }^{17}$ Si veda Savić, M., Temporalni kondicional $u$ italijanskom jeziku, Belgrado 1966. 
Il lessico carducciano conserva una grandissima parte del tradizionale vocabolario «poetico». Sintomatica la parola avello, che compare con eccezionale frequenza (RSM 9, 28, 41, 90, PV 338, 348, 382, 392, 408, J 153, 224, 226, LG 332, 352, 366, GE 9, 73, 99, RN 173, 223, 236, 287, 340, RR 203, ecc); e così acciaro (RSM 29, PV 356, J 222, LG 338, GE 73, RN 298, 304, RR 238), aere (RSM 31, 94, PV 355, 369, J 14, LG 295, RN 172, 260, OB 40, 127, RR 223, 248), alma (RSM 8, PV 366, 432, LG 306, OB 122, RR 203, 233), augello (RSM 46, PV 397, 398, 439, LG 292, 320, 353, GE 13, 99, RN 203, 221, 368, OB 49, 74, 99, RR 184, 188, 216), desio (o disio, desire, ecc.: RSM 8, 20, 49, PV 349, 366, J 211, LG 292, 320, 337, 372, GE 3, 28, 128, RN 185, 229, 233, 271, OB 41, 115, RR 179, 228), di (GE 27, 42, 46, 63, 91, 110, RN 166, 198, 215, 273, 358, OB 29, 42, 71, RR 218, 233, ecc.), duolo (RSM 22, 83, 101, PV 334, 375, LG 294), guardo (LG 303, RR 177), il giuro (LG 315, GE 79), nappo (RSM 59, PV 441, RN 194, 340), periglio (RSM 98, LG 325, RR 215), polve (PV 349, 420, J 118, LG 370, RR 185), prence (RSM 97, J 112, 236, RN 183, RR 177), i rai (RSM 25, RN 192, OB 12, 71), rege (J 192, GE 45, RN 169, OB 16), speme o spene (RSM 24, 87, 90, J 208, LG 282, RN 190, 358), tenzone (RSM 9, J 210, LG 307, 363), i vanni (PV 346, 368, 380, 388, LG 284, RN 224), verno (LG 353, RN 192, OB 59, 109, RR 234, 254), ecc.; gli aggettivi almo (RSM 19, PV 464, GE 19, OB 118, RR 249), avito (PV 450, LG 342, GE 51, OB 27, RR 194), imo (RSM 94, PV 394, 414), pargolo o parbolo (LG 301, 316, 351, GE 100, RN 201, 357, OB 62, 105, 108, 123, RR 212), picciolo (RSM 13, 87, PV 464, J 106, GE 109, OB 60, 67, RR 244), ratto (PV 372, 398, 418, LG 340), reo o rio (nel senso di «cattivo«: RSM 8, 17, 28, 36, 52, PV 335, 366, 463, J 204, 212, LG 284, 317, GE 8, 106, RN 225, 273, OB 16, 102, RR 217), veglio (RSM 97, LG 372, GE 32, RN 167), ecc.; i verbi desiare o disiare (RSM 83, LG 358, OB 12, 93), favellare (RSM 53, PV 342, 355, 461, OB 45, 80), fiedere (RSM 22, J 237, LG 348, RN 213, OB 108, RR 195), giovare (nel senso di «piacere»: LG 354, RN 287), guatare (RSM 52, 75, PV 435, 465, J 4, LG 320, RN 272), mirare (RSM 49, PV 338, OB 26, 116, 122, RR 223), nodrire (RSM 11, PV 356, 409), redire (RSM 12, 22, 85, PV 344, LG 294, 366, RN 249), ecc.; altre parole come anco (RSM 47, 94, PV 464, J 203, LG 281, 372, GE 27), cotanto (RSM 42, J 151, LG 299, OB 86), indarno (RSM 31, PV 386, 418), niuno (RSM 39, J 116, 200, LG 303, GE 30, 80, RN 169), ognora ( $P V$ 373, LG 321, RN 340, RR 209), pria ( $P V$ 342, 355, J 207, LG 345, GE 4, RN 168, 303, RR 192, 200, 254), quinci (RSM 9, 33, PV 450, J 152, 206), ecc.

Particolarmente caratteristici del C. sono i latinismi (e grecismi). Lasciando da parte quelli da lui introdotti, che sono già stati messi in rilievo nella Storia del Migliorini (p. 682-683, 731-732) e altrove, daremo piuttosto qualche esempio dei latinismi tradizionali, che il nostro ha potuto mutuare dai poeti delle epoche precedenti: angue (PV 335, GE 20), ara (LG 315, GE 32, OB 89), arbore (LG 283, RN 173, 348), carme ( $\mathrm{J}$ 35, 154, LG 286, GE 130, RN 165, 260, OB 24, 123, RR 183), cervice 
(RSM 18, PV 394, 404, OB 42), clade (RSM 31, J 199, LG 338), cuna (PV 335, 356, 366, LG 315, RN 224, RR 214), delubro (GE 4, OB 10, 27), fòro (GE 67, RN 184, OB 40), imago (RSM 10, 53, 92, PV 451, LG 294), lavacro (PV 347, 402, OB 48, RR 199), nato «figlio» (RSM 93, PV 397, 459, GE 51), nembo (LG 373, GE 36, OB 27, 75), plaustro (LG 298, OB 23), procella (PV 399, 410, 452, GE 8), talamo (RSM 100, PV 338, J 229, LG 315, GE 10, 109, RN 252, OB 31, 114, RR 211), e, non ultimo, ]'emblematico pate (LG 342, 366, GE 49, 70, RN 167, 182, 319, OB 67, RR 184, 222); inoltre aggettivi come antiquo (RSM 28, 53, PV 455), diro (RSM 25 28, 92, PV 451, OB 26, 131), egro (RSM 55, J 195, RR 234), igneo (PV 350, RN 234, OB 120, RR 126), miro (RSM 46, OB 20, RR 203), negro (PV 388, 426, LG 291, $\mathrm{RN} 189,214, \mathrm{RR} 224$ ), virente ( $\mathrm{PV}$ 355, OB 98), ecc.

Nelle poesie giovanili sono frequenti gli aggettivi composti di tipo grecizzante, presi certamente dalla poesia settecentesca: armipotente (PV 337), pariosplendenti (380), alicandenti (382), nerovestito (447, J 228). Anche più tardi troviamo isolatamente biancovestite (RN 320) o il lungochiomato lombardo (OB 45).

Mentre la base aulica del vocabolario rimane sostanzialmente immutata lungo l'arco della creazione carducciana, al fianco dei vocaboli «blasonati» vengono gradualmente a porsi parole della lingua quotidiana, inconcepibili nella poesia italiana tradizionale. È questa la novità più significativa del Carducci, anche se in questo riguardo egli è preceduto dal Berchet e superato da alcuni' suoi contemporanei (in primo luogo dagli scapigliati). Non importano in questo senso le espressioni crudamente realistiche, a volte volgari, normali nella poesia satirica (soprattutto nei Giambi ed epodi: chiasso, puzzo, schiaffo, capatappi, papero, mulo, gonzo, cavolo, carogna, ma anche nelle Rime nuove: caff'e latte, cabolfiori, dissenteria, birichino, ghiottone, vigliacco, vecchie ciancianti, ecc): si tratta, com'è noto, di una tradizione plurisecolare, particolarmente forte in Toscana. Interessano invece le parole comuni, «neutrali», che per il loro significato o anche per la loro forma corrispondono esattamente a una parola del lessico aulico. Il Carducci non abbandona quest'ultima a favore della nuova, ma adopera entrambe come sinonimi di valore più o meno uguale, ai fini di una maggiore varietà lessicale. Così, mentre permane (come si è visto sopra) l'uso di augello, appare nelle poesie più tarde anche il normale uccello (RN 231, 238, 260, 331, OB 45, 101, 127, 134, RR 221, 231); si ha perfino uccelli e augelli nella stessa poesia ( $\mathrm{RN}$ 266). Accanto a dì, appare costantemente giorno (GE 19, 42, 57, RN 160, 192, 201, 217, 247, 302, OB 14, 38, 73, RR 184, 219, 242), accanto ad avello, tomba (GE 32, 36, 46, 51, 76, RN 175, 229, 258, 345, OB 36, 66, 111 RR 176, 221), accanto a speme, speranza (RN 159, 201, 235, $\mathrm{OB} 36,63,127)$, accanto ad arbore, albero (RN 187, 211, 231, 266, OB 95), accanto a guardo, sguardo (GE 78, RN 332, OB 81, 131), accanto a picciolo, piccolo (RN 195, 303, OB 30,121) e piccolino (RN 227, 330). Nubola (GE 95, OB 28, 43, 79, 132) prende posto accanto a nube, e un verso le 
contiene entrambe: ha baciato / la nube, e ha detto - Nubola bianca, t'apri (RR 188). Anima e usignuolo si affiancano ad alma e rusignolo. Accanto a sembiante, viso, volto, il poeta non esita a usare faccia (GE 62, RN 201, 232, 275, 309, OB 96, RR 178, 219); c'è nappo e calice, ma anche il semplice bicchiere (GE 112, 207, $\mathrm{OB} 93,137)$, canto e carme, ma anche canzone (OB 59, 82, 104, 134, RR 177, 193, 231). Anche all'infuori delle poesie satiriche appaiono vocaboli prosaici quali piazza (GE 110, RN 234, 278, OB 134, RR 222), vacca (OB 12, 114), ritmo (OB 78, RR 253), diabolo (RN 194, 302, RR 241). Al fianco di innumerevoli fanciulle e donzelle, si affaccia timidamente una ragazza $(\mathrm{RN} 329)$, ed altre categorie di persone sono designate con il loro vero nome: bimbo (GE 86, RN 284), bambino (GE 111, RN 349), moglie (OB 28, RR 215), prete (GE 16, 27, 40, 82, 106, RN 225, 265, 325), papa (GE 111, RN 194, OB 43), soldato (RN 278, 360), contadino (RN 313). Si veda anche Elwert, cit., 125-126.

Alla fine di questa rassegna, ci sembra di poter condividere, sebbene con qualche riserva, il giudizio del De Lollis, secondo cui «il Carducci non riuscì, anzi neppure aspirò ... a una poesia tutta storia, cioè tutta cose, rifatte in una coscienza moderna, la quale poesia si spogliasse dell'involucro della forma tradizionale... II Carducci puramente lirico, dal sonetto al Bope ... alle Odi barbare ... è sempre e tutto della maniera tradizionale» (op. cit., 116-117). Accettiamo, cioè, il concetto del «fondo aulico», sul quale si sovrappongono diversi elementi di modernità, ma che rimane pur sempre la base e il cardine della lingua carducciana. Una tale definizione ci sembra più vicina alla verità che non le troppo ottimistiche valutazioni del Borlenghi, che parla di un felice connubio fra «una lingua comune e moderna, concreta e pregnante e viva» e «il linguaggio poetico tradizionale» (op. cit., p. CXI), oppure dell'Elwert, che definisce lo stile del $\mathrm{C}$. «continuatore bensì della tradizione classica, o meglio, aulica, della poesia italiana; ma ... ben diverso già dalla lingua aulica della poesia amorosa del Settecento e anche dal classicismo pariniano e manzoniano e dal classicismo... del Leopardi» (op. cit., 127). Questa diversità e questa modernità esistono indubbiamente nella poesia carducciana e sono, come abbiamo detto, fra le prime impressioni che si ricavano dalla letura; ma, come crediamo di aver dimostrato con la nostra breve indagine, non risiedono nella lingua. Sono soprattutto la fonetica e la morfologia a far pendere la bilancia dalla parte dell'antico. Se si volesse compilare una «grammatica italiana» in base ai vari cittade, chieggo, meco, giacean, vommene, moriro, vorrien, il feo, se 'n giva ecc. elencati sopra, tutte le regole di questa grammatica sarebbero valide per la lingua del Leopardi, del Parini e anche di poeti più antichi, mentre si dimostrerebbero assolutamente inapplicabili, per esempio, alla lingua giornalistica dell'epoca carducciana. Le forme arcaiche sono frequenti soprattutto nelle poesie giovanili, alquanto attenuate negli Juoenilia (che pure riprendono gran parte del materiale delle Rime di San Miniato), nei Levia gravia, Giambi ed epodi e Rime nuove, e regrediscono 
ancora nella poesia «barbara» delle ultime due raccolte. Ma questo succede non perché il C. abbia deciso di modernizzare la sua morfologia, bensì perché il suo stile si è fatto più vivo, più vario e originale, quindi meno dipendente dall'imitazione di modelli classici. Con l'allargarsi del già vasto panorama della lingua carducciana, le forme antiquate vengono a occupare una parte proporzionalmente più piccola, ${ }^{18}$, ma esse non sono mai ripudiate in quanto arcaiche, e tanto meno soppiantate dagli equivalenti moderni. La sovrastruttura diventa sempse più importante e più complessa, ma il fondamento aulico non cambia.

Più o meno identiche conclusioni valgono per il lessico. Diventando progressivamente più audace nelle sue ricerche creative, il poeta adopera sempre più le parole comuni, tipo uccello, ma senza rinunciare a quelle auliche tipo augello. Si tratta di due livelli separati da una minima differenza stilistica, che nel C. possono benissimo coesistere. Non siamo ancora nel mondo del Pascoli, che abbandonerà augello fin dagli inizi e troverà presto troppo generico anche uccello, sostituendolo con precise indicazioni ornitologiche; o del Gozzano, che userà augello soltanto tra ironiche virgolette. Il fatto è che per il $\mathrm{C}$. la modernità o l'arcaicità di una parola non ha importanza; la sua poesia non si muove sulla linea «antico-nuovo», ma in altre dimensioni. Il suo bisogno di «parole pellegrine» si appaga in vari modi: talvolta con arcaismi assoluti, antiquati anche rispetto al vocabolario aulico normale della sua poesia, altre volte con parole moderne, usate in poesia per la prima volta, e ancora più spesso con parole che non sono né vecchie né nuove, ma semplicemente inedite, si tratti di coniazioni sue ${ }^{19}$ o di latinismi adoperati soltanto da lui, che per questo fatto - e anche per la stessa natura del latino, eterno «serbatoio lessicale» dell'italiano - rimangono fuori tempo. In breve, abbiamo a che fare con un poeta che non si chiude alle parole dell'uso, ma che non sente alcun bisogno di rinnovare il suo linguaggio nel senso in cui avevano voluto farlo i romantici e in cui poi l'avrebbero fatto Pascoli e i crepuscolari. Ed è anche questa una ragione per cui, mentre tanti poeti alla fine dell'Otto e all'inizio del Novecento tenteranno di imitarlo nelle loro poesie giovanili, la storia della letteratura italiana non registrerà nessun «carducciano» all'infuori del Carducci stesso.

18 Una situazione analoga, ma molto più spiccata, si avrà nel D’Annunzio, nella cui poesia gli arcaismi tradizionali passano quasi inosservati nella massa di arcaismi «nuovi» da lui stesso scoperti nei dizionari e nei testi antichi, affiancati da latinismi, grecismi, tecnicismi e ogni altra specie di «parole rare e preziose».

19 Si veda Devoto, op. cit. 248, per alcuni di questi neologismi (usati, è vero, soprattutto nella prosa del C.): tra $\mathrm{i}$ «preziosismii il Devoto include anche le grafie del tipo co'l e il gruppo consonantico di conscienza, instituto. V. anche Bertoni, op. cit. 91, per gli arcaismi che il C. trovò nei classici italiani e che «parvero bizzarrie e stranezze al Fanfani». 


\section{Rezime}

Giosue Carducci je jedini veliki pesnik iz perioda kada je italijanska poezija napuštala tradicionalni jezik, iskovan još u vreme Petrarke, i postepeno ga zamenjivala živim govornim jezikom svoga doba. Otuda je važno utvrditi koliki udeo u Carduccijerom jeziku imaju nasledjeni poetski arhaizmi. Pregled tekstova pokazuje da je C. najkonzervativniji $\mathfrak{u}$ morfologiji, gde uveliko upotrebljava, na primer, stare oblike ličnih zamenica, enklitičke zamenice posle glagola u indikativu i proklitičke ispred imperativa, zastarele nastavke u prezentu glagola I konjugacije, imperfektu II i III konjugacije, 3. licu jednine i množine preterita, kondicionalu itd., kao i arhaične oblike glagola essere, fare, andare i drugih. Manje takvih pojava ima u fonetici, gde se ne mogu uvel jasno razlikovati arhaizmi od toskanizama. Red reči je u osnovi linearan i blizak proznom stilu, mada u mladalačkim pesmama ima još dosta tradicionalnih inverzija i mada se neke od njih sreću i kasnije. Za rečnik je karakteristično da C. zadržava takoreći sve stare poetske izraze, ali im u kasnijem periodu svog stvaralaštva priključuje i savremene reči, tako da paralelno upotrebljava npr. augello i uccello, nube i nubola, pargolo i bambino i sl. Latinizmi imaju dvostruku vrednost, pošto su neki od njih prenzeti od italijanskih klasika, dok su drugi inovacije onog tipa koji će doći još do mnogo većeg izražaja u delu D’Annunzij̄a. Opšti zaključak je da kod Carduccija tradicionalni jezik čini osnovu, na koju se, naročito na leksičkom polju, nadovezuju elementi proznog i govornog jezika, ali (za razliku od Pascolija i drugih novijih pesnika) stari oblici se ne odbacuju i nema svesne želje za modernizacijom. 